Disclosure of Interests: Evangelia Passia: None declared, Marijn Vis Grant/ research support from: Novartis, Pfizer - grant/research support, Consultant of: AbbVie, Celgene Corporation, Eli Lilly, Novartis, Pfizer - consultant, Laura C Coates: None declared, Anushka Soni Grant/research support from: OxfordUCB prize fellowship, Speakers bureau: Janssen and Abbvie, Ilja Tchetverikov: None declared, Andreas Gerards: None declared, Lindy-Anne Korswagen: None declared, Marc R Kok Grant/research support from: BMS and Novartis, Consultant of: Novartis and Galapagos, Wiebo van der Graaff: None declared, Josien Veris-van Dieren: None declared, Natasja Denissen: None declared, F. Fodili: None declared, M. Starmans: None declared, Yvonne Goekoop-Ruiterman: None declared, M. van Oosterhout: None declared, Jolanda Luime: None declared

DOI: 10.1136/annrheumdis-2020-eular.3461

\section{OP0058-PARE MY SJÖGREN'S DIARY: AN ONLINE PATIENT PORTAL FOR PATIENT LED SJÖGREN'S SYNDROME RESEARCH}

$\underline{\text { C. Burns }}^{1}$, H. Breitmeyer ${ }^{1}$, L. Cowley ${ }^{2}$, S. Govind ${ }^{3}$, W. F. Ng${ }^{4}$, T. F. Hiemstra ${ }^{2,5}$ ${ }^{1}$ British Sjögren's Syndrome Association, Cambridge, United Kingdom; ${ }^{2}$ Patient Led Research Hub, Cambridge, United Kingdom; ${ }^{3}$ Cambridge Clinical Trials Unit, Cambridge, United Kingdom; ${ }^{4}$ Newcastle University, Newcastle, United Kingdom; ${ }^{5}$ University of Cambridge, Medicine, Cambridge, United Kingdom

Background: Sjögren's Syndrome is a chronic autoimmune disease affecting the exocrine glands accompanied by variable extra-glandular manifestations Symptom flares, including dry eyes, dry mouth, dry skin, fatigue, myalgia and arthralgia, are frequent. Many of these symptoms have a considerable impact on quality of life, but are variable, sensitive to external factors, and difficult to measure objectively.

Clinical research on Sjögren's Syndrome is advancing with the help of patient registries: an array of clinical data is collected and available for approved studies. However, many of these registries focus on collecting clinical information and often fail to capture the diverse patient experience adequately. Thus, there is an unmet need for an online 'patient portal', secure and encrypted with the capability of interacting with existing registries, while also patient-facing to encourage active involvement in research and personal healthcare. Patient demand for this resource was highlighted in 2017 when the Cambridgeshire regional support group of the British Sjögren's Syndrome Association (BSSA) contacted the Patient Led Research Hub (PLRH). The PLRH provides research expertise to co-produce research ideas with patient organisations ${ }^{1}$; the PLRH and Cambridgeshire group have since secured funding and initiated work on this project with the Cambridge Clinical Trials Unit.

Objectives: Develop 'My Sjögren's Diary' a cross-platform patient portal to:

1.Act as an interactive tool to help patients manage their healthcare needs and aid communication with healthcare providers.

2.Function as a research platform, enabling patients to consent to contact, as well as support home-based data entry, allowing real-time capture of symptom scores and ensuring ease of participation for patients. Link with the UK Primary Sjögren's Syndrome Registry and NHS Digital to provide complementary clinical datasets.

Methods: The PLRH has coordinated a team of rheumatologists, database programmers, patients and family members to develop My Sjögren's Diary. Regular meetings, national surveys and correspondence with patients ensures the project remains relevant to patient needs, while collaborating with rheumatologists ensures the database is reliable, valid and of benefit to clinical care. Workshops hosted at key stages of database development have allowed both patients and rheumatologists to direct and refine My Sjögren's Diary. A prototype was presented at the 2019 BSSA Annual Conference before further improvements and beta release.

Results: A beta version of My Sjögren's Diary enabling BSSA members to track their medication and symptoms is now active. Feedback will be incorporated into the final version before it is publicly available to Sjögren's Syndrome patients. Further funding is required to develop the research platform.

Conclusion: My Sjögren's Diary encourages equal partnership between patients, clinicians and researchers. It presents a unique opportunity for comprehensive analysis of Sjögren's Syndrome and associated health utilities. Research participation is not mandatory, encouraging all patients to have an active role in personal healthcare management.

References:

[1] Mader LB, Harris T, Kläger S, Wilkinson IB, Hiemstra TF. Inverting the patient involvement paradigm: defining patient led research. Research Involvement and Engagement 4, 21 (2018).

Acknowledgments: Thank you to Cambridgeshire and North Eastern BSSA regional support groups for ongoing review of the portal's usability, function and design. Thank you to BSSA for project funding, and to the Cambridge Clinical Trials Unit and University of Cambridge Medical Library for providing workshop facilities.

Disclosure of Interests: None declared

DOI: 10.1136/annrheumdis-2020-eular.138

\section{Artificial intelligence and machine learning in imag- ing of rheumatology: Are we ready?}

\section{OP0059 AUTOSCORA: DEEP LEARNING TO AUTOMATE SCORING OF RADIOGRAPHIC PROGRESSION IN RHEUMATOID ARTHRITIS}

T. Deimel ${ }^{1}$, D. Aletaha ${ }^{1}$, G. Langs ${ }^{1} .{ }^{1}$ Medical University of Vienna, Vienna, Austria

Background: The prevention of joint destruction is an important goal in the man agement of rheumatoid arthritis (RA) and a key endpoint in drug trials. To quantify structural damage in radiographs, standardized scoring systems ${ }^{1}$, such as the Sharp/van der Heijde (SvdH) score ${ }^{2}$, which separately assesses joint space narrowing (JSN) and erosions, have been developed. However, application of these scores is time-consuming, requires specially trained staff, and results are subject to considerable intra- and inter-reader variability ${ }^{1}$. This makes their application poorly feasible in clinical practice and limits their reliability in clinical trials. Objectives: We aim to develop a fully automated deep learning-based scoring system of radiographic progression in RA to facilitate the introduction of quantitative joint damage assessment into daily clinical practice and circumvent interreader variability in clinical trials.

Methods: 5191 hand radiographs and their corresponding SvdH JSN scores from 640 adult patients with RA without visible joint surgery were extracted from the picture archive of a large tertiary hospital. The dataset was split, on a patien level, into training (2207 images/270 patients), validation (1150/133), and test (1834/237) sets. Joints were automatically localized using a particular deep learning model $^{3}$ which utilizes the local appearance of joints combined with information on the spatial relationship between joints. Small regions of interest (ROI) were automatically extracted around each joint. Finally, different deep learning architectures were trained on the extracted ROls using the manually assigned SvdH JSN scores as ground truth (Fig. 1). The best models were chosen based on their performance on the validation set. Their ability to assign the correct SvdH JSN scores to ROls was assessed using the unseen data of the test set.

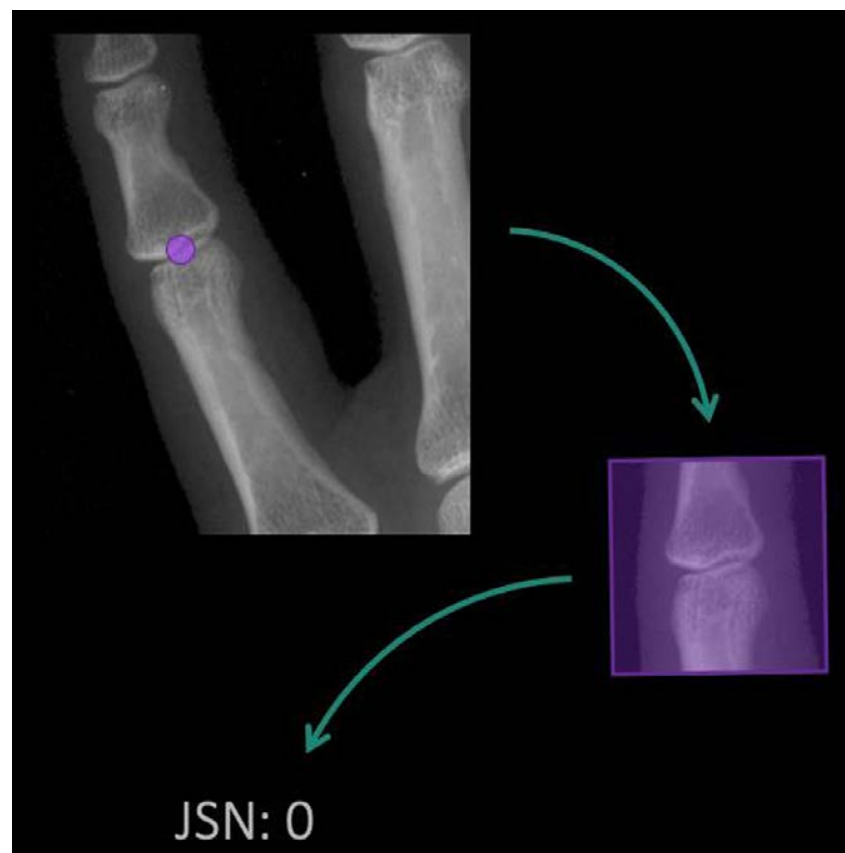

Fig. 1. 3-step approach to automated scoring: joint localization, ROI extraction, JSN scoring

Results: ROI extraction was successful in $96 \%$ of joints, meaning that all struc tures were visible and joints were not malrotated by more than 30 degrees. For JSN scoring, modifications of the VGG16 ${ }^{4}$ architecture seemed to outperform adaptations of DenseNet ${ }^{5}$. The mean obtained accuracy (i.e., the percentage of 